\title{
Distances to convex sets
}

\author{
by \\ Antonio S. Granero and Marcos SÁnchez (Madrid)
}

\begin{abstract}
If $X$ is a Banach space and $C$ a convex subset of $X^{*}$, we investigate whether the distance $\widehat{d}\left(\overline{\mathrm{co}}^{w^{*}}(K), C\right):=\sup \left\{\inf \{\|k-c\|: c \in C\}: k \in \overline{\mathrm{co}}^{w^{*}}(K)\right\}$ from $\overline{\mathrm{co}}^{w^{*}}(K)$ to $C$ is $M$-controlled by the distance $\widehat{d}(K, C)$ (that is, if $\widehat{d}\left(\overline{\mathrm{co}}{ }^{w^{*}}(K), C\right) \leq$ $M \widehat{d}(K, C)$ for some $1 \leq M<\infty)$, when $K$ is any weak ${ }^{*}$-compact subset of $X^{*}$. We prove, for example, that: (i) $C$ has 3 -control if $C$ contains no copy of the basis of $\ell_{1}$ (c); (ii) $C$ has 1-control when $C \subset Y \subset X^{*}$ and $Y$ is a subspace with weak ${ }^{*}$-angelic closed dual unit ball $B\left(Y^{*}\right)$; (iii) if $C$ is a convex subset of $X$ and $X$ is considered canonically embedded into its bidual $X^{* *}$, then $C$ has 5 -control inside $X^{* *}$, in general, and 2-control when $K \cap C$ is weak* ${ }^{*}$ dense in $C$.
\end{abstract}

1. Introduction. If $X$ is a Banach space and $C$ a convex subset of $X^{*}$, we investigate in this paper whether the distance $\widehat{d}\left(\overline{\mathrm{co}}^{w^{*}}(K), C\right):=$ $\sup \left\{\inf \{\|k-c\|: c \in C\}: k \in \overline{\mathrm{co}} w^{*}(K)\right\}$ from $\overline{\mathrm{co}} w^{*}(K)$ to $C$ is controlled by the distance $\widehat{d}(K, C)$, that is, if $\widehat{d}\left(\overline{\mathrm{co}} w^{*}(K), C\right) \leq M \widehat{d}(K, C)$ for some constant $1 \leq M<\infty$ independent of $K$, where $K$ is any weak ${ }^{*}$-compact subset of $X^{*}$.

When $C$ is a subspace of $X$, the control of $C$ inside the bidual $X^{* *}$ of $X$ has been studied in [10]-[13]. Actually the results obtained in those papers extend the classical Krein-Shmul'yan theorem. This theorem, in terms of distances, states the following (see [8, p. 29]): if $X$ is a Banach space and $K$ a weak*-compact subset of $X^{* *}$ such that $\widehat{d}(K, X)=0$ (that is, $K$ is a weak-compact subset of $X)$, then $\widehat{d}\left(\overline{\mathrm{co}} w^{*}(K), X\right)=0$, that is, $\overline{\mathrm{co}} w^{*}(K) \subset X$ and so $\overline{\mathrm{co}} w^{*}(K)$ is a weak-compact subset of $X$ and $\overline{\mathrm{co}^{*}} w^{*}(K)=\overline{\mathrm{co}}(K)$. Thus, looking at the Krein-Shmul'yan theorem in terms of distances, it is natural to ask the following: if $K$ is a weak*-compact subset of $X^{* *}$, does the equality $\widehat{d}\left(\overline{\mathrm{co}} w^{*}(K), X\right)=\widehat{d}(K, X)$ always hold? The answer to this question is negative. Actually, in [11] and [12] are constructed two weak ${ }^{*}$-compact

2000 Mathematics Subject Classification: 46B20, 46B26.

Key words and phrases: convex sets, distances, Krein-Shmul'yan theorem.

Supported in part by grant DGICYT MTM2005-00082, grant UCM-910346 and grant UCM-BSCH PR27/05-14045. 
subsets $K_{1}, K_{2}$ of a bidual Banach space $X^{* *}$ such that: (i) $K_{1} \cap X$ is weak*dense in $K_{1}, \widehat{d}\left(K_{1}, X\right)=1 / 2$ and $\widehat{d}\left(\overline{\mathrm{co}}^{w^{*}}\left(K_{1}\right), X\right)=1$; (ii) $\widehat{d}\left(K_{2}, X\right)=1 / 3$ and $\widehat{d}\left(\overline{\mathrm{CO}}^{w^{*}}\left(K_{2}\right), X\right)=1$.

Thus, in general, a Banach space $X$ fails to have 1-control inside its bidual $X^{* *}$. However, it could be true that every Banach space $X$ has $M$ control inside $X^{* *}, M$ being a universal constant greater than 1 . So, we can ask the following question: does there exist a universal constant $1<M<\infty$ such that $\widehat{d}\left(\overline{\mathrm{co}} w^{*}(K), X\right) \leq M \widehat{d}(K, X)$ for every weak*-compact subset $K$ of $X^{* *}$ and every Banach space $X$ ? The answer to this question is affirmative. In [11] the following result is proved, which extends the Krein-Shmul'yan theorem: if $K$ is a weak*-compact subset of $X^{* *}$ and $Z$ a subspace of $X$, then

$$
\widehat{d}\left(\overline{\mathrm{co}} w^{*}(K), Z\right) \leq 5 \widehat{d}(K, Z) ;
$$

moreover, if $Z \cap K$ is weak ${ }^{*}$-dense in $K$, then

$$
\widehat{d}\left(\overline{\mathrm{co}} w^{*}(K), Z\right) \leq 2 \widehat{d}(K, Z) .
$$

When $H$ is a normal countably compact space and we look at the Banach space $Z=C(H)$ of continuous real functions on $H$ as a subspace of $\ell_{\infty}(H)$, then the distances $\widehat{d}\left(\overline{\mathrm{co}} w^{*}(K), Z\right)$ and $\widehat{d}(K, Z)$ behave analogously, $K$ being any weak ${ }^{*}$-compact subset of $\ell_{\infty}(H)$ (see [3], [14]). So, in view of these results we have: (i) the smallest value $M_{0}$ of the universal constant of the extension of the Krein-Shmul'yan theorem satisfies $3 \leq M_{0} \leq 5$; (ii) for the category of weak*-compact subsets $K$ of $X^{* *}$ such that $Z \cap K$ is weak*-dense in $K, Z$ being a subspace of $X$, the value $M=2$ is optimal.

The purpose of this paper is to go a step further and investigate the control of $\widehat{d}\left(\overline{\mathrm{co}}^{w^{*}}(K), C\right)$ by $\widehat{d}(K, C)$ when $C$ is a convex subset of a dual Banach space $X^{*}$ and $K$ is a weak*-compact subset of $X^{*}$. The behavior of $\widehat{d}\left(\overline{\mathrm{co}}^{w^{*}}(K), C\right)$ with respect to $\widehat{d}(K, C)$ varies. If $C$ is a weak ${ }^{*}$-closed convex subset of $X^{*}$, it is very easy to see that $\widehat{d}\left(\overline{\mathrm{co}}^{w^{*}}(K), C\right)=\widehat{d}(K, C)$. However, if $C \subset X^{*}$ is not weak*-closed, all situations are possible. In any case, as we will see later, the control of $C$ inside $X^{*}$ and the existence in $C$ of a copy of the basis of $\ell_{1}(\mathfrak{c})$ are closely connected.

The paper is organized as follows. In Section 2 we study the control of convex subsets $C$ of a Banach space $X$ inside $X^{* *}$. The results and constants obtained are similar to the ones obtained when $C$ is a subspace of $X$.

In Section 3 we deal with the relation between the existence in $C$ of a copy of the basis of $\ell_{1}(\mathfrak{c})$ and the control of $C$ inside a dual Banach space $X^{*}$. We prove that every convex subset $C$ of $X^{*}$ has 3 -control inside $X^{*}$ whenever $C$ contains no copy of the basis of $\ell_{1}(\mathfrak{c})$. Moreover, $\overline{\mathrm{co}}^{w^{*}}(K)=\overline{\mathrm{co}}(K)$ for every weak*-compact subset $K$ of $X^{*}$ that contains no copy of that basis. 
Section 4 is devoted to the study of the control of a convex subset $C$ inside a dual Banach space $X^{*}$ when $C$ is contained in a subspace $Y$ of $X^{*}$ with weak*-angelic closed dual unit ball $B\left(Y^{*}\right)$. This case is particularly favorable because always $\widehat{d}\left(\overline{\mathrm{co}} w^{*}(K), C\right)=\widehat{d}(K, C)$ for every weak*-compact subset $K$ of $X^{*}$.

Our notation is standard. If $A$ and $I$ are sets, $a \in A^{I}$ and $i \in I$ then $a_{i}$ (or $a(i)$ ) denotes the $i$ th coordinate of $a$ and $\pi_{i}: A^{I} \rightarrow A$ the $i$ th projection mapping such that $\pi_{i}(a)=a_{i} .|I|$ is the cardinality of $I$ and $\mathfrak{c}:=|\mathbb{R}|$. $\beta I$ denotes the Stone-Cech compactification of $I$ (for $I$ endowed with the discrete topology) and $I^{*}:=\beta I \backslash I$. If $f: I \rightarrow \mathbb{R}$ is a bounded function, then $\check{f} \in C(\beta I)$ is the Stone-Cech continuous extension of $f$ to $\beta I$.

We shall consider only Banach spaces over the real field. If $X$ is a Banach space, let $B(a ; r):=\{x \in X:\|x-a\| \leq r\}$ be the closed ball with center at $a \in X$ and radius $r \geq 0 . B(X)$ and $S(X)$ will be the closed unit ball and unit sphere of $X$, respectively, and $X^{*}$ its topological dual. If $A$ is a subset of $X$, then $[A]$ and $\overline{[A]}$ denote the linear hull and the closed linear hull of $A$, respectively. A subset $A$ of the Banach space $X$ is said to contain a copy of the basis of $\ell_{1}(\mathfrak{c})$ if $A$ contains a family of vectors $\left\{a_{i}: i<\mathfrak{c}\right\}$ which is equivalent to the canonical basis of $\ell_{1}(\mathfrak{c})$. The weak ${ }^{*}$-topology of the dual Banach space $X^{*}$ is denoted by $w^{*}$ and the weak topology of $X$ by $w$. If $C$ is a convex subset of $X^{*}$, for $x^{*} \in X^{*}$ and $A \subset X^{*}$, let $d\left(x^{*}, C\right)=\inf \left\{\left\|x^{*}-c\right\|: c \in C\right\}$ be the distance from $x^{*}$ to $C$, and $\widehat{d}(A, C)=\sup \{d(a, C): a \in A\}$ the distance from $A$ to $C$. $\operatorname{co}(A)$ denotes the convex closure of the set $A, \overline{\mathrm{co}}(A)$ is the $\|\cdot\|$-closure of $\operatorname{co}(A)$ and $\overline{\mathrm{co}} w^{*}(A)$ the $w^{*}$-closure of $\operatorname{co}(A)$. Given $1 \leq M<\infty$, a convex subset $C$ of $X^{*}$ is said to have $M$-control inside $X^{*}$ if $\widehat{d}\left(\overline{\mathrm{co}}^{w^{*}}(K), C\right) \leq$ $M \widehat{d}(K, C)$ for every $w^{*}$-compact subset $K$ of $X^{*}$; and $C$ is said to have control inside $X^{*}$ if $C$ has $M$-control inside $X^{*}$ for some constant $1 \leq M<\infty$.

If $K$ is a $w^{*}$-compact subset of a dual Banach space $X^{*}$ and $\mu$ a Radon Borel probability on $K$, then $r(\mu)$ will denote the barycenter of $\mu$ (see [6, p. 115]). Recall that: (i) $r(\mu) \in \overline{\mathrm{co}}^{w^{*}}(K)$; (ii) $x^{*} \in \overline{\mathrm{co}}^{w^{*}}(K)$ if and only if there exists a Radon Borel probability $\mu$ on $K$ such that $r(\mu)=x^{*}$; (iii) $r(\mu)(x)=\int_{K} x^{*}(x) d \mu\left(x^{*}\right)$ for all $x \in X$.

2. The control of convex subsets of $X$ inside $X^{* *}$. Convex subsets of a bidual Banach space $X^{* *}$, in general, fail to have control inside $X^{* *}$. For example, if $X$ is a Banach space such that $X^{*}$ contains a copy of $\ell_{1}$, then there exists a $w^{*}$-compact subset $H$ of $X^{* *}$ such that $\widehat{d}\left(\overline{\mathrm{co}}^{w^{*}}(H), \overline{\mathrm{co}}(H)\right)>0$ (see [15]). However, when we restrict ourselves to the convex subsets $C$ of the Banach space $X$, we will see in this section that there exists control inside $X^{* *}$. We begin with the calculation of the distance $d(x, C)$ when $C$ is a convex subset of a Banach space $X$ and $x \in X$. 
Lemma 2.1. Let $X$ be a Banach space, $C$ a convex subset of $X$ and $x \in X$. Then

$$
d(x, C)=\sup _{\varphi \in S\left(X^{*}\right)} \inf \{|\varphi(x-c)|: c \in C\} .
$$

Moreover, if $x \notin \bar{C}$, then even $d(x, C)=\sup _{\varphi \in S\left(X^{*}\right)} \inf \varphi(x-C)$.

Proof. If we assume that $x \notin \bar{C}$, the proof is a simple application of the Banach separation theorem. If $x \in \bar{C}$, then for every $\varphi \in S\left(X^{*}\right)$ we have $\inf \{|\varphi(x-c)|: c \in C\}=0$, whence

$$
d(x, C)=0=\sup _{\varphi \in S\left(X^{*}\right)} \inf \{|\varphi(x-c)|: c \in C\} .
$$

The following lemmas are basic for the proofs of next propositions.

Lemma 2.2. Let $X$ be a Banach space and $D$ a convex subset of $X$. Then for every $z \in \bar{D}^{w^{*}} \subset X^{* *}$ we have

$$
d(z, D) \leq 2 d(z, X) .
$$

Proof. Suppose that $d(z, D)>2 d(z, X)$. Then

(i) for some $a>0$ we have $d(z, D)>2 a>2 d(z, X)$,

(ii) there exists a vector $w \in X$ such that $\|w-z\|<a$ (because $d(z, X)$ $<a$ ) and so $d(w, D)>a$ (otherwise, if $d(w, D) \leq a$, we would get $d(z, D) \leq\|w-z\|+d(w, D)<2 a$, a contradiction $).$

Since $d(w, D)>a$, by Lemma 2.1 there exists $x^{*} \in S\left(X^{*}\right)$ such that $\inf \left\{x^{*}(w-d): d \in D\right\}>a$. Let $\left\{d_{i}\right\}_{i \in I} \subset D$ be a net such that $d_{i} \stackrel{w^{*}}{\rightarrow} z$. Then $w-d_{i} \stackrel{w^{*}}{\rightarrow} w-z$ and so $x^{*}\left(w-d_{i}\right) \rightarrow x^{*}(w-z)$. Hence $x^{*}(w-z)>a$ and so $\|w-z\|>a$, a contradiction. Thus, we get $d(z, D) \leq 2 d(z, X)$.

Lemma 2.3. Let $X$ be a Banach space, $C$ a convex subset of $X^{*}, K$ a $w^{*}$-compact subset of $X^{*}$ and assume there exist $a, b>0$ such that

$$
\widehat{d}(K, C)<a<b<\widehat{d}\left(\overline{\mathrm{co}} w^{*}(K), C\right) .
$$

Then there exist $z_{0} \in \overline{\mathrm{co}} w^{*}(K)$ and $\psi \in S\left(X^{* *}\right)$ with $\inf \psi\left(z_{0}-C\right)>b$ such that, if $\mu$ is a Radon probability on $K$ with barycenter $r(\mu)=z_{0}$ and $H=\operatorname{supp}(\mu)$, for every $w^{*}$-open subset $V$ of $X^{*}$ with $V \cap H \neq \emptyset$ there exists $\xi \in \overline{\mathrm{co}}^{w^{*}}(V \cap H)$ such that $\inf \psi(\xi-C)>b$.

Proof. Without loss of generality, we suppose that $K \subset B\left(X^{*}\right)$. Choose $z \in \overline{\mathrm{co}} w^{*}(K)$ such that $d(z, C)>b$. By Lemma 2.1 there exists $\psi \in S\left(X^{* *}\right)$ such that $\inf \psi(z-C)>b+\varepsilon$ for some $\varepsilon>0$, that is, $\psi(z)>b+\varepsilon+$ $\sup \psi(C)$. By the Bishop-Phelps theorem, there exists a vector $\phi \in S\left(X^{* *}\right)$ with $\|\psi-\phi\| \leq \varepsilon / 4$ such that $\phi$ attains its maximum on $\overline{\mathrm{CO}}^{w^{*}}(K)$ at some point $z_{0} \in \overline{\mathrm{co}} w^{*}(K)$. So 


$$
\begin{aligned}
\phi\left(z_{0}\right) & \geq \phi(z)=\psi(z)+(\phi-\psi)(z) \\
& >\sup \psi(C)+b+\varepsilon-\frac{1}{4} \varepsilon=\sup \psi(C)+b+\frac{3}{4} \varepsilon,
\end{aligned}
$$

whence we get

$\psi\left(z_{0}\right)=\phi\left(z_{0}\right)+(\psi-\phi)\left(z_{0}\right)>\sup \psi(C)+b+\frac{3}{4} \varepsilon-\frac{1}{4} \varepsilon=\sup \psi(C)+b+\frac{1}{2} \varepsilon$, that is,

$$
\inf \psi\left(z_{0}-C\right)>b+\frac{1}{2} \varepsilon
$$

Thus $d\left(z_{0}, C\right)>b+\frac{1}{2} \varepsilon$ and so $z_{0} \notin \bar{C}$ and $z_{0} \notin K$ (because $\left.\widehat{d}(K, C)<a<b\right)$. Let $\mu$ be a Radon probability on $K$ with $r(\mu)=z_{0}$ and let $H:=\operatorname{supp}(\mu)$. Assume that there exists a $w^{*}$-open subset $V$ of $X^{*}$ with $V \cap H \neq \emptyset$ such that $\inf \psi(\xi-C) \leq b$ (that is, $\psi(\xi) \leq b+\sup \psi(C))$ for every $\xi \in \overline{\mathrm{co}}^{w^{*}}(V \cap H)$. Let $\mu_{1}=\mu \nmid V \cap H$ denote the restriction of $\mu$ to $V \cap H$, that is, $\mu_{1}(B)=$ $\mu(B \cap V \cap H)$ for every Borel subset $B \subset K$. Let $\mu_{2}:=\mu-\mu_{1}$. Observe that $\mu_{1}$ and $\mu_{2}$ are positive measures such that

(i) $\mu_{1} \neq 0$, because $\emptyset \neq V \cap H=V \cap \operatorname{supp}(\mu)$,

(ii) $\mu_{2} \neq 0$ because, if we assume $\mu_{2}=0$ (that is, $\mu=\mu_{1}=\mu \nmid V \cap H$ ), then $z_{0}=r(\mu) \in \overline{\mathrm{co}}^{w^{*}}(V \cap H)$ and so $\inf \psi\left(z_{0}-C\right) \leq b$, a contradiction to $(2.2)$.

Thus, we have the decomposition $\mu=\mu_{1}+\mu_{2}$ such that $1=\|\mu\|=\left\|\mu_{1}\right\|+$ $\left\|\mu_{2}\right\|$ with $\left\|\mu_{1}\right\| \neq 0 \neq\left\|\mu_{2}\right\|$. So, we can write

$$
z_{0}=r(\mu)=\left\|\mu_{1}\right\| \cdot r\left(\frac{\mu_{1}}{\left\|\mu_{1}\right\|}\right)+\left\|\mu_{2}\right\| \cdot r\left(\frac{\mu_{2}}{\left\|\mu_{2}\right\|}\right) .
$$

Since $r\left(\mu_{1} /\left\|\mu_{1}\right\|\right) \in \overline{\mathrm{co}}^{w^{*}}(V \cap H)$, we have $\psi\left(r\left(\mu_{1} /\left\|\mu_{1}\right\|\right)\right) \leq b+\sup \psi(C)$ by hypothesis. Hence $\phi\left(r\left(\mu_{1} /\left\|\mu_{1}\right\|\right)\right) \leq b+\frac{1}{4} \varepsilon+\sup \psi(C)$ (because $\|\psi-\phi\| \leq$ $\varepsilon / 4)$. Thus, taking into account that $r\left(\mu_{2} /\left\|\mu_{2}\right\|\right) \in \overline{\mathrm{co}}^{*}(K), \phi\left(r\left(\mu_{2} /\left\|\mu_{2}\right\|\right)\right)$ $\leq \phi\left(z_{0}\right)$ and $(2.1)$, we get

$$
\begin{aligned}
\phi\left(z_{0}\right) & =\left\|\mu_{1}\right\| \phi\left(r\left(\frac{\mu_{1}}{\left\|\mu_{1}\right\|}\right)\right)+\left\|\mu_{2}\right\| \phi\left(r\left(\frac{\mu_{2}}{\left\|\mu_{2}\right\|}\right)\right) \\
& \leq\left\|\mu_{1}\right\|\left(b+\frac{1}{4} \varepsilon+\sup \psi(C)\right)+\left\|\mu_{2}\right\| \phi\left(z_{0}\right) \\
& <\left\|\mu_{1}\right\| \phi\left(z_{0}\right)+\left\|\mu_{2}\right\| \phi\left(z_{0}\right)=\phi\left(z_{0}\right),
\end{aligned}
$$

a contradiction which completes the proof.

Proposition 2.4. Let $X$ be a Banach space, $C$ a convex subset of $X$ and $K a w^{*}$-compact subset of $X^{* *}$. Then

$$
\widehat{d}\left(\overline{\mathrm{co}}^{w^{*}}(K), C\right) \leq 5 \widehat{d}(K, C) .
$$

Proof. Without loss of generality, we assume that $0 \in C$. Suppose that the statement is not true, so there exists a $w^{*}$-compact subset $K$ of $X^{* *}$ and $a, b>0$ such that

$$
\widehat{d}\left(\overline{\mathrm{co}}^{w^{*}}(K), C\right)>b>5 a>5 \widehat{d}(K, C) .
$$


From Lemma 2.3 we have the following:

FACT. There exists a functional $\psi \in S\left(X^{* * *}\right)$ and a $w^{*}$-compact subset $\emptyset \neq H \subset K$ such that for every $w^{*}$-open subset $V$ with $V \cap H \neq \emptyset$ there exists $\xi \in \overline{\mathrm{co}}^{w^{*}}(V \cap H)$ with $\inf \psi(\xi-C)>b$.

Now we carry out the following construction step by step:

SteP 1. Let $D_{0}=\{0\}$. Applying the Fact to the $w^{*}$-open subset $V_{0}:=$ $X^{* *}$, we choose a vector $\xi_{1} \in \overline{\mathrm{co}} w^{*}(H)$ such that $\inf \psi\left(\xi_{1}-C\right)>b$. So, $\psi\left(\xi_{1}\right)>b+\sup \psi\left(D_{0}\right)=b$. As $B\left(X^{*}\right)$ is $w^{*}$-dense in $B\left(X^{* * *}\right)$, there exists $x_{1}^{*} \in S\left(X^{*}\right)$ such that $x_{1}^{*}\left(\xi_{1}\right)>b+\max x_{1}^{*}\left(D_{0}\right)=b$. Let $W_{1}:=\left\{u \in X^{* *}\right.$ : $\left.\left\langle u, x_{1}^{*}\right\rangle>b+\max x_{1}^{*}\left(D_{0}\right)=b\right\}$. Clearly, $W_{1}$ is a $w^{*}$-open halfspace of $X^{* *}$ such that $\xi_{1} \in W_{1} \cap \overline{\mathrm{co}} w^{*}(H)$. Thus, $W_{1} \cap H \neq \emptyset$ and so we can find a vector $\eta_{1} \in W_{1} \cap H$. Since $d\left(\eta_{1}, C\right)<a$, we have a decomposition $\eta_{1}=\eta_{1}^{1}+\eta_{1}^{2}$ such that $\eta_{1}^{1} \in C$ and $\eta_{1}^{2} \in a B\left(X^{* *}\right)$.

SteP 2. Let $D_{1}=\left\{\eta_{1}^{1}\right\} \cup D_{0} \subset C$ and $V_{1}:=W_{1} \cap V_{0}=W_{1}$. As $V_{1}$ is a $w^{*}$-open subset with $V_{1} \cap H \neq \emptyset$, by the Fact there exists a vector $\xi_{2} \in \overline{\mathrm{co}}^{w^{*}}\left(V_{1} \cap H\right)$ such that $\inf \psi\left(\xi_{2}-C\right)>b$, and also inf $\psi\left(\xi_{2}-D_{1}\right) \geq$ $\inf \psi\left(\xi_{2}-C\right)>b$ because $D_{1} \subset C$. Since $D_{1}$ is finite and $\min \psi\left(\xi_{2}-D_{1}\right)>b$, there exists a vector $x_{2}^{*} \in S\left(X^{*}\right)$ such that $\min x_{2}^{*}\left(\xi_{2}-D_{1}\right)>b$, that is, $x_{2}^{*}\left(\xi_{2}\right)>b+\max x_{2}^{*}\left(D_{1}\right)$. Let $W_{2}:=\left\{u \in X^{* *}:\left\langle u, x_{2}^{*}\right\rangle>b+\max x_{2}^{*}\left(D_{1}\right)\right\}$. Clearly, $W_{2}$ is a $w^{*}$-open halfspace of $X^{* *}$ such that $\xi_{2} \in W_{2} \cap \overline{\mathrm{co}}^{w^{*}}\left(V_{1} \cap H\right)$. Thus $W_{2} \cap V_{1} \cap H \neq \emptyset$ and we can find $\eta_{2} \in W_{2} \cap V_{1} \cap H$. So, $x_{2}^{*}\left(\eta_{2}\right)>$ $b+\max x_{2}^{*}\left(D_{1}\right)$, that is, $\min x_{2}^{*}\left(\eta_{2}-D_{1}\right)>b$. Moreover, $\min x_{1}^{*}\left(\eta_{2}-D_{0}\right)>b$ because $\eta_{2} \in V_{1}$. Since $d\left(\eta_{2}, C\right)<a$, we have a decomposition $\eta_{2}=\eta_{2}^{1}+\eta_{2}^{2}$ such that $\eta_{2}^{1} \in C$ and $\eta_{2}^{2} \in a B\left(X^{* *}\right)$.

By iteration, we get sequences $\left\{x_{n}^{*}\right\}_{n \geq 1} \subset S\left(X^{*}\right),\left\{\eta_{k}\right\}_{k \geq 1} \subset H, D_{k}=$ $\left\{\eta_{k}^{1}\right\} \cup D_{k-1}$ with $\eta_{k}=\eta_{k}^{1}+\eta_{k}^{2}, \eta_{k}^{1} \in C$ and $\eta_{k}^{2} \in a B\left(X^{* *}\right), k \geq 1$, such that $\min x_{i}^{*}\left(\eta_{k}-D_{i-1}\right)>b$ for every $k \geq i$.

Let $D=\overline{\mathrm{co}}\left(\bigcup_{k \geq 1} D_{k}\right) \subset \bar{C}$ and

$$
K_{1}={\overline{\left\{\eta_{i}^{1}: i \geq 1\right\}}}^{w^{*}} \subset\left(K+a B\left(X^{* *}\right)\right) \cap \bar{D}^{w^{*}} .
$$

Let $\eta_{0}$ be a $w^{*}$-cluster point of $\left\{\eta_{k}\right\}_{k \geq 1}$.

Claim 1. $d\left(\eta_{0}, D\right)<5 a$.

Indeed, clearly $\eta_{0} \in H \cap\left(K_{1}+a B\left(X^{* *}\right)\right)$. Observe that:

(i) Since $K_{1} \subset K+a B\left(X^{* *}\right)$, we get $\widehat{d}\left(K_{1}, C\right) \leq \widehat{d}(K, C)+a<2 a$.

(ii) Since $K_{1} \subset \bar{D}^{w^{*}}$, by Lemma 2.2 we get $\widehat{d}\left(K_{1}, D\right) \leq 2 \widehat{d}\left(K_{1}, X\right) \leq$ $2 \widehat{d}\left(K_{1}, C\right)<4 a$.

Thus, as $\eta_{0} \in K_{1}+a B\left(X^{* *}\right)$, we finally get $d\left(\eta_{0}, D\right)<5 a$.

Claim 2. $d\left(\eta_{0}, D\right) \geq b$. 
Indeed, let $\phi \in B\left(X^{* * *}\right)$ be a $w^{*}$-cluster point of $\left\{x_{n}^{*}\right\}_{n \geq 1}$. Since we have $\min x_{n}^{*}\left(\eta_{k}-D_{n-1}\right)>b$ for every $k \geq n$, it follows that $\min x_{n}^{*}\left(\eta_{0}-D_{n-1}\right) \geq b$ for all $n \geq 1$. Hence $\inf \phi\left(\eta_{0}-D\right) \geq b$ and so $d\left(\eta_{0}, D\right) \geq b$ by Lemma 2.1.

Since $b>5 a$, we get a contradiction that completes the proof.

Proposition 2.5. Let $X$ be a Banach space, $C \subset X$ a convex subset of $X$, and $K$ a $w^{*}$-compact subset of $X^{* *}$ such that $K \cap C$ is $w^{*}$-dense in $K$. Then $\widehat{d}\left(\overline{\mathrm{co}} w^{*}(K), C\right) \leq 2 \widehat{d}(K, C)$.

Proof. Suppose that $\widehat{d}\left(\overline{\mathrm{co}}^{w^{*}}(K), C\right)>b>2 a>2 \widehat{d}(K, C)$ for some $a, b>0$. We follow the proof of Proposition 2.4 with the following changes. As $C \cap K$ is $w^{*}$-dense in $K$ and $V_{k} \cap H \neq \emptyset, k \geq 0$, it follows that $V_{k} \cap C \cap K \neq \emptyset$ for all $k \geq 0$. Thus, we choose $\eta_{k} \in V_{k} \cap K \cap C, k \geq 1$, and put $\eta_{k}^{1}=\eta_{k}$ and $\eta_{k}^{2}=0$. Hence, now $K_{1}={\overline{\left\{\eta_{k}^{1}: k \geq 1\right\}}}^{w^{*}}={\overline{\left\{\eta_{k}: k \geq 1\right\}}}^{w^{*}}$ satisfies $K_{1} \subset K$ and so $\widehat{d}\left(K_{1}, C\right) \leq \widehat{d}(K, C)<a$, whence $\widehat{d}\left(K_{1}, D\right)<2 a$. Finally, every $w^{*}$-cluster point $\eta_{0}$ of $\left\{\eta_{k}: k \geq 1\right\}$ satisfies $\eta_{0} \in K_{1}, d\left(\eta_{0}, D\right)<2 a$ and $d\left(\eta_{0}, D\right) \geq b$, a contradiction.

REMARK 2.6. In Proposition 2.4 we have proved that there exists a constant $M$ such that $1 \leq M \leq 5$ and $\widehat{d}\left(\overline{\mathrm{co}}^{w^{*}}(K), C\right) \leq M \widehat{d}(K, C)$ for every Banach space $X$, every convex subset $C$ of $X$ and every $w^{*}$-compact subset $K$ of $X^{* *}$. It is an open problem what is the best value $M_{0}$ of this constant, but $3 \leq M_{0} \leq 5$ by the results of [12]. Concerning the constant of Proposition 2.5, the value $M=2$ is optimal by [12].

3. Distances to convex subsets of dual Banach spaces. Let $X$ be a Banach space, $C$ a convex subset of $X^{*}$, and $W$ a $w^{*}$-compact subset of $X^{*}$. In this section we study whether the distance $\widehat{d}\left(\overline{\mathrm{co}} w^{*}(W), C\right)$ is controlled by the distance $\widehat{d}(W, C)$. The following proposition is an elementary result.

Proposition 3.1. Let $C$ be a $w^{*}$-closed convex subset of the dual Banach space $X^{*}$. Then $C$ has 1-control inside $X^{*}$, that is, for every $w^{*}$-compact subset $W$ of $X^{*}$ we have $\widehat{d}\left(\overline{\mathrm{co}}^{w *}(W), C\right)=\widehat{d}(W, C)$.

Proof. Let $W$ be a $w^{*}$-compact subset of $X^{*}$ and let $\widehat{d}(W, C)=$ : $a$. Fix a point $w_{0} \in \overline{\mathrm{co}}^{w^{*}}(W)$ and a number $\varepsilon>0$; we prove that $d\left(w_{0}, C\right) \leq a+\varepsilon$. Let $\left\{w_{\alpha}: \alpha \in \mathcal{A}\right\} \subset \operatorname{co}(W)$ be a net such that $w_{\alpha} \stackrel{w^{*}}{\rightarrow} w_{0}$ for $\alpha \in \mathcal{A}$. Since $\widehat{d}(\operatorname{co}(W), C)=\widehat{d}(W, C)$, for each $\alpha \in \mathcal{A}$ we can choose $z_{\alpha} \in C$ such that $\left\|w_{\alpha}-z_{\alpha}\right\|<a+\varepsilon$. So, the net $\left\{w_{\alpha}-z_{\alpha}: \alpha \in \mathcal{A}\right\}$ is inside the ball $(a+\varepsilon) B\left(X^{*}\right)$, which is a $w^{*}$-compact subset. Thus, by passing to a subnet if necessary, we can suppose that $w_{\alpha}-z_{\alpha} \stackrel{w^{*}}{\rightarrow} u_{0}$ for some $u_{0} \in(a+\varepsilon) B\left(X^{*}\right)$. Hence, we get $z_{\alpha}=w_{\alpha}-\left(w_{\alpha}-z_{\alpha}\right) \stackrel{w^{*}}{\rightarrow} w_{0}-u_{0}$ and so $w_{0}-u_{0}=: z_{0} \in C$, because $C$ is $w^{*}$-closed. Therefore, we can write $w_{0}=z_{0}+u_{0}$ with $z_{0} \in C$ 
and $u_{0} \in(a+\varepsilon) B\left(X^{*}\right)$, that is, $d\left(w_{0}, C\right) \leq a+\varepsilon$. As $\varepsilon>0$ is arbitrarily small, we conclude that $d\left(w_{0}, C\right) \leq a=\widehat{d}(W, C)$. So, $\widehat{d}\left(\overline{\mathrm{co}} w^{*}(W), C\right)=\widehat{d}(W, C)$.

The following result is a consequence of Lemma 2.3.

LEMMA 3.2. Let $X$ be a Banach space and $K$ a $w^{*}$-compact subset of $X^{*}$ such that $\widehat{d}\left(\overline{\mathrm{co}} w^{*}(K), \overline{\mathrm{co}}(K)\right)>d>0$. Then there exist $r_{0} \in \mathbb{R}, z_{0} \in \overline{\mathrm{co}} w^{*}(K)$ and $\psi \in S\left(X^{* *}\right)$ with $\psi\left(z_{0}\right)>r_{0}+d$ and $\psi(k)<r_{0}$ for all $k \in K$, and such that, if $\mu$ is a Radon probability on $K$ with barycenter $r(\mu)=z_{0}$ and $H=\operatorname{supp}(\mu)$, then:

(i) for every $w^{*}$-open subset $V \subset X^{*}$ with $V \cap H \neq \emptyset$, there exist $\xi \in$ $\overline{\mathrm{CO}} w^{*}(V \cap H)$ such that $\psi(\xi)>r_{0}+d$,

(ii) there exist a sequence $\left\{x_{n}: n \geq 1\right\} \subset B(X)$ and, for every pair of disjoint subsets $M, N$ of $\mathbb{N}$, a point $\eta_{M, N} \in H$ such that $\eta_{M, N}\left(x_{m}\right) \geq r_{0}+d, \quad \forall m \in M, \quad$ and $\eta_{M, N}\left(x_{n}\right) \leq r_{0}, \forall n \in N$.

Proof. Find $\varepsilon>0$ such that $\widehat{d}\left(\overline{\mathrm{co}} w^{*}(K), \overline{\mathrm{co}}(K)\right)>d+\varepsilon>0=\widehat{d}(K, \overline{\mathrm{co}}(K))$. By Lemma 2.3 there exist $z_{0} \in \overline{\mathrm{co}}^{w^{*}}(K)$ and $\psi \in S\left(X^{* *}\right)$ such that $\inf \psi\left(z_{0}-\overline{\mathrm{co}}(K)\right)>d+\varepsilon$, that is,

$$
\psi\left(z_{0}\right)>\sup \psi(\overline{\operatorname{co}}(K))+d+\varepsilon \geq \sup \psi(K)+\varepsilon+d .
$$

So, if $r_{0}:=\sup \psi(K)+\varepsilon$, then $\psi\left(z_{0}\right)>r_{0}+d$ and $\psi(k)<r_{0}$ for all $k \in K$. Let $\mu$ be a Radon Borel probability on $K$ with $r(\mu)=z_{0}$ and let $H:=\operatorname{supp}(\mu)$.

Claim. For every $w^{*}$-open subset $V$ of $X^{*}$ with $V \cap H \neq \emptyset$ there exist $\xi \in \overline{\mathrm{cO}}^{w^{*}}(V \cap H)$ and $\eta \in \overline{\mathrm{co}}(V \cap H) \subset \overline{\mathrm{co}}^{w^{*}}(V \cap H)$ such that $\psi(\xi)>r_{0}+d$ and $\psi(\eta)<r_{0}$.

Indeed, by Lemma 2.3 there exists $\xi \in \overline{\mathrm{co}}^{w^{*}}(V \cap H)$ with $\inf \psi(\xi-\overline{\mathrm{co}}(K))$ $>d+\varepsilon$, that is, $\psi(\xi)>r_{0}+d$. On the other hand, as $\psi(k)<r_{0}$ for all $k \in K$, we have $\psi(\eta)<r_{0}$ for every $\eta \in \operatorname{co}(V \cap H)$.

Thus, by the Claim and the proof of [15, Lemma 2] we can find a sequence $\left\{x_{n}: n \geq 1\right\} \subset S(X)$ such that, if we define

$$
A_{n}=\left\{\xi \in H: \xi\left(x_{n}\right)>r_{0}+d\right\}, \quad B_{n}=\left\{\eta \in H: \eta\left(x_{n}\right)<r_{0}\right\}, \quad \forall n \geq 1,
$$

then, for every pair of disjoint finite subsets $M, N$ of $\mathbb{N}$, the $w^{*}$-open subset $V(M, N):=\left(\bigcap_{m \in M} A_{m}\right) \cap\left(\bigcap_{n \in N} B_{n}\right)$ of $H$ is nonempty. In particular,

$$
\emptyset \neq V(M, N) \subset\left(\bigcap_{m \in M} \bar{A}_{m}^{w^{*}}\right) \cap\left(\bigcap_{n \in N} \bar{B}_{n}^{w^{*}}\right) \subset H .
$$

Since $H$ is a $w^{*}$-compact subset, we conclude that for every pair of disjoint (finite or infinite) subsets $M, N$ of $\mathbb{N}$,

$$
\emptyset \neq\left(\bigcap_{m \in M} \bar{A}_{m}^{w^{*}}\right) \cap\left(\bigcap_{n \in N} \bar{B}_{n}^{w^{*}}\right) \subset H .
$$


Since $\bar{A}_{m}^{w^{*}} \subset\left\{\xi \in H: \xi\left(x_{m}\right) \geq r_{0}+d\right\}$ and $\bar{B}_{n}^{w^{*}} \subset\left\{\eta \in H: \eta\left(x_{n}\right) \leq r_{0}\right\}$, we finally deduce that for every pair of disjoint (finite or infinite) subsets $M, N$ of $\mathbb{N}$ there exists $\eta_{M, N} \in H$ such that

$\eta_{M, N}\left(x_{m}\right) \geq r_{0}+d, \quad \forall m \in M, \quad$ and $\quad \eta_{M, N}\left(x_{n}\right) \leq r_{0}, \quad \forall n \in N$.

Definition 3.3. If $X$ is a Banach space, a subset $\mathcal{F}$ of $X^{*}$ is said to be a $w^{*}-\mathbb{N}$-family of width $d>0$ if $\mathcal{F}$ is bounded and has the form

$$
\mathcal{F}=\left\{\eta_{M, N}: M, N \text { disjoint subsets of } \mathbb{N}\right\}
$$

so that there exist two sequences $\left\{r_{m}: m \geq 1\right\} \subset \mathbb{R}$ and $\left\{x_{m}: m \geq 1\right\} \subset$ $B(X)$ such that for every pair of disjoint subsets $M, N$ of $\mathbb{N}$ we have

$$
\eta_{M, N}\left(x_{m}\right) \geq r_{m}+d, \quad \forall m \in M, \quad \text { and } \quad \eta_{M, N}\left(x_{n}\right) \leq r_{n}, \quad \forall n \in N .
$$

Moreover, if $r_{m}=r_{0}$ for all $m \geq 1$, we say that $\mathcal{F}$ is a uniform $w^{*}-\mathbb{N}$-family in $X^{*}$. We say that $A \subset X^{*}$ has a $w^{*}-\mathbb{N}$-family if there exists a $w^{*}-\mathbb{N}$-family $\mathcal{F} \subset A$.

REMARK 3.4. (0) If $Z$ is a set, a family $\left(A_{i}, B_{i}\right)_{i \in I}$ of pairs of nonempty subsets of $Z$ is said to be an independent family if $A_{i} \cap B_{i}=\emptyset$ for all $i \in I$, and for every finite nonempty subset $F \subset I$ we have $\bigcap_{i \in F} \varepsilon_{i} A_{i} \neq \emptyset$, where $\varepsilon_{i}=$ $\pm 1,(+1) A_{i}=A_{i}$ and $(-1) A_{i}=B_{i}$. In $\mathbb{N}$ there exists an independent family $\left(M_{i}, N_{i}\right)_{i<\mathfrak{c}}$ of cardinality $\mathfrak{c}$. Indeed, since $\beta \mathbb{N}$ is an extremally disconnected compact Hausdorff space with weight $\mathrm{w}(\beta \mathbb{N})=\mathfrak{c}$ (see [21, p. 76]), by the Balcar-Franěk theorem (see [2], [7, p. 120]) there exists a continuous onto mapping $f: \beta \mathbb{N} \rightarrow\{0,1\}^{\mathfrak{c}}$. Let $\pi_{i}:\{0,1\}^{\mathfrak{c}} \rightarrow\{0,1\}, i<\mathfrak{c}$, be the projection onto the $i$-factor $\{0,1\}$ and put $M_{i}:=\left(\pi_{i} \circ f\right)^{-1}(1) \cap \mathbb{N}$ and $N_{i}:=\left(\pi_{i} \circ\right.$ $f)^{-1}(0) \cap \mathbb{N}$. Clearly, $\left\{\left(M_{i}, N_{i}\right): i<\mathfrak{c}\right\}$ is an independent family in $\mathbb{N}$.

(1) If $\left(M_{i}, N_{i}\right)_{i<\mathfrak{c}}$ is an independent family in $\mathbb{N}$ of cardinality $\mathfrak{c}$ and $\mathcal{F}=$ $\left\{\eta_{M, N}: M, N\right.$ disjoint subsets of $\left.\mathbb{N}\right\}$ is a $w^{*}-\mathbb{N}$-family in the dual Banach space $X^{*}$, then a standard argument (see [8, p. 206]) proves that the family $\left\{\eta_{M_{i}, N_{i}}: i<\mathfrak{c}\right\}$ is equivalent to the basis of $\ell_{1}(\mathfrak{c})$. Moreover, the same argument shows that the sequence $\left\{x_{n}: n \geq 1\right\} \subset B(X)$ associated to $\mathcal{F}$ is equivalent to the basis of $\ell_{1}$.

(2) So, if a dual Banach space $X^{*}$ has a $w^{*}$-N - -family, then $X$ has an isomorphic copy of $\ell_{1}$. And vice versa, if $X$ has a copy of $\ell_{1}$, then $X^{*}$ contains a $w^{*}$-N-family. Indeed, let $i: \ell_{1} \rightarrow X$ be an isomorphism between $\ell_{1}$ and $i\left(\ell_{1}\right)$, and $i^{*}: X^{*} \rightarrow \ell_{\infty}$ its adjoint operator, which is a quotient mapping such that $B\left(\ell_{\infty}\right) \subset i^{*}\left(\left\|i^{-1}\right\| B\left(X^{*}\right)\right)$. For each pair $M, N$ of disjoint subsets of $\mathbb{N}$ choose $\eta_{M, N} \in\left\|i^{-1}\right\| B\left(X^{*}\right)$ such that $i^{*}\left(\eta_{M, N}\right)=\mathbf{1}_{M}-\mathbf{1}_{N}$. Then $\left\{\eta_{M, N}: M, N\right.$ disjoint subsets of $\left.\mathbb{N}\right\}$ is a $w^{*}$ - $\mathbb{N}$-family in $X^{*}$.

(3) Let $\mathcal{F}=\left\{\eta_{M, N}: M, N\right.$ disjoint subsets of $\left.\mathbb{N}\right\}$ be a $w^{*}$ - $\mathbb{N}$-family of width $\delta>0$ in a dual Banach space $X^{*}$, associated to the sequences $\left\{r_{m}: m \geq 1\right\} \subset \mathbb{R}$ and $\left\{x_{m}: m \geq 1\right\} \subset B(X)$. Then for every $0<$ 
$\gamma<\delta$ there exists an infinite subset $\mathbb{N}_{\gamma} \subset \mathbb{N}$ such that $\mathcal{F}_{\gamma}:=\left\{\eta_{M, N}\right.$ : $M, N$ disjoint subsets of $\left.\mathbb{N}_{\gamma}\right\}$ is a uniform $w^{*}$-N -family of width $\gamma>0$ associated to the sequence $\left\{x_{m}: m \in \mathbb{N}_{\gamma}\right\} \subset B(X)$ and some number $r_{0} \in \mathbb{R}$. Indeed, since the sequence $\left\{r_{m}: m \geq 1\right\} \subset \mathbb{R}$ is bounded, there exists some $r_{0} \in \mathbb{R}$ such that $\mathbb{N}_{\gamma}:=\left\{m \in \mathbb{N}: r_{0}+\eta-\delta \leq r_{m} \leq r_{0}\right\}$ is infinite. Now, it is easy to see that $\mathcal{F}_{\gamma}:=\left\{\eta_{M, N}: M, N\right.$ disjoint subsets of $\left.\mathbb{N}_{\gamma}\right\}$ is a uniform $w^{*}$ - $\mathbb{N}$-family of width $\gamma>0$ associated to $r_{0}$ and the sequence $\left\{x_{m}: m \in \mathbb{N}_{\gamma}\right\} \subset B(X)$.

(4) It is worth mentioning (and easy to see) that, if $A$ is a subset of $X^{*}$, then $A$ has a $w^{*}-\mathbb{N}$-family if and only if $\bar{A}$ does.

Proposition 3.5. Let $X$ be a Banach space.

(1) If $K$ is a $w^{*}$-compact subset of $X^{*}$ such that $K$ fails to have a $w^{*}$ $\mathbb{N}$-family (in particular, if $K$ contains no copy of the basis of $\ell_{1}(\mathfrak{c})$ ), then $\overline{\mathrm{co}}^{w^{*}}(K)=\overline{\mathrm{co}}(K)$.

(2) If $C$ is a convex subset of $X^{*}$ that fails to have a $w^{*}-\mathbb{N}$-family (in particular, if $C$ contains no copy of the basis of $\left.\ell_{1}(\mathfrak{c})\right)$, then $C$ has 3 -control inside $X^{*}$, that is, for every $w^{*}$-compact subset $K$ of $X^{*}$ we have $\widehat{d}\left(\overline{\mathrm{co}} w^{*}(K), C\right) \leq 3 \widehat{d}(K, C)$.

Proof. (1) Otherwise, there exists $d>0$ such that $\widehat{d}\left(\overline{\mathrm{CO}}^{w^{*}}(K), \overline{\mathrm{co}}(K)\right)>$ $d>0$. By Lemma 3.2 there exist $\left\{x_{n}: n \geq 1\right\} \subset B(X), r_{0} \in \mathbb{R}$ and, for every pair of disjoint subsets $M, N$ of $\mathbb{N}$, a vector $\eta_{M, N} \in K$ such that

$\eta_{M, N}\left(x_{m}\right) \geq r_{0}+d, \quad \forall m \in M, \quad$ and $\quad \eta_{M, N}\left(x_{n}\right) \leq r_{0}, \quad \forall n \in \mathbb{N}$.

Thus there exists a $w^{*}-\mathbb{N}$-family in $K$, a contradiction.

(2) Suppose that $C$ fails to have 3 -control inside $X^{*}$. Then there exist a $w^{*}$-compact subset $K$ of $X^{*}$ and $a, b>0$ such that $\widehat{d}\left(\overline{\mathrm{co}}^{w^{*}}(K), C\right)>b>$ $3 a>3 \widehat{d}(K, C)$. So, as $\widehat{d}(\overline{\mathrm{co}}(K), C)=\widehat{d}(K, C)<a$, we have $\widehat{d}\left(\overline{\mathrm{co}}^{w^{*}}(K), \overline{\mathrm{co}}(K)\right)$ $>b-a>0$. By Lemma 3.2 there exist $r_{0} \in \mathbb{R},\left\{x_{n}: n \geq 1\right\} \subset B(X)$ and, for every pair of disjoint subsets $M, N$ of $\mathbb{N}$, a vector $\eta_{M, N} \in K$ such that

$\eta_{M, N}\left(x_{m}\right) \geq r_{0}+b-a, \quad \forall m \in M, \quad$ and $\quad \eta_{M, N}\left(x_{n}\right) \leq r_{0}, \quad \forall n \in N$. As $\widehat{d}(K, C)<a$, for each pair of disjoint subsets $M, N$ of $\mathbb{N}$ there is $z_{M, N} \in C$ so that $\left\|z_{M, N}-\eta_{M, N}\right\|<a$. Thus, the family $\left\{z_{M, N}: M, N\right.$ disjoint subsets of $\mathbb{N}\}$ is bounded and satisfies

$z_{M, N}\left(x_{m}\right) \geq r_{0}+b-2 a, \quad \forall m \in M, \quad$ and $\quad z_{M, N}\left(x_{n}\right) \leq r_{0}+a, \quad \forall n \in \mathbb{N}$. Since $r_{0}+b-2 a=r_{0}+a+(b-3 a)>r_{0}+a$, the set $\left\{z_{M, N}: M, N\right.$ disjoint subsets of $\mathbb{N}\}$ is a $w^{*}-\mathbb{N}$-family in $C$, a contradiction.

Remark 3.6. For a convex subset $C$ of a dual Banach space $X^{*}$, the statements " $C$ has 3 -control inside $X^{*}$ " and " $C$ contains no $w^{*}-\mathbb{N}$-family" are not equivalent, in general. For example, if $C:=B\left(\ell_{\infty}\right)$, then $C$ has a $w^{*}$ $\mathbb{N}$-family (this is trivial), and also $C$ has 1 -control (and so 3 -control) inside 
$\ell_{\infty}$ because $C$ is $w^{*}$-closed (see Proposition 3.1). Concerning the statement " $C$ contains no copy of the basis of $\ell_{1}(\mathfrak{c})$ ", it can be characterized as follows.

Proposition 3.7. Let $X$ be a Banach space and $C$ a convex subset of $X^{*}$. The following statements are equivalent:

(i) $C$ contains no copy of the basis of $\ell_{1}(\mathfrak{c})$.

(ii) $C$ has universal 3-control, that is, if $\overline{[C]}$ is (isomorphic to) a subspace of some dual Banach space $V^{*}$, then $C$ has 3 -control inside $V^{*}$.

(iii) $C$ has universal control, that is, if $\overline{[C]}$ is (isomorphic to) a subspace of some dual Banach space $V^{*}$, then $C$ has control inside $V^{*}$.

Proof. (i) $\Rightarrow$ (ii) follows from Proposition 3.5 , and (ii) $\Rightarrow$ (iii) is obvious.

(iii) $\Rightarrow$ (i). Suppose that $C$ contains a copy $K$ of the basis of $\ell_{1}(\mathfrak{c})$ and let $Z:=[C]$. By the proof of [13, Prop. 3] there exists a closed subspace $V$ of $Z^{*}$ norming on $Z$ such that $K$ is $\sigma(Z, V)$-compact but $\overline{\mathrm{co}}^{\sigma(Z, V)}(K)$ is not $\sigma(Z, V)$-compact. Let $i: Z \rightarrow V^{*}$ be the canonical embedding such that $i(z)(v)=\langle v, z\rangle$ for all $z \in Z$ and $v \in V$. Clearly, $i$ is a norm-isomorphism between $Z$ and $i(Z)$. Moreover, $i:(Z, \sigma(Z, V)) \rightarrow\left(i(Z), w^{*}\right)$ is also an isomorphism. Then $i(K)$ is a $w^{*}$-compact subset of $V^{*}$ such that $i(K) \subset$ $i(C)$. Since $\overline{\mathrm{co}} \sigma(Z, V)(K)$ is not $\sigma(Z, V)$-compact in $(Z, \sigma(Z, V))$, necessarily $\overline{\mathrm{co}}^{w^{*}}(i(K)) \backslash i(Z) \neq \emptyset$ and so $\widehat{d}\left(\overline{\mathrm{co}} w^{*}(i(K)), i(C)\right)>0$. Thus $i(C)$ does not have control inside $V^{*}$, a contradiction to (iii).

A result of Talagrand [20] allows us to prove the following corollary:

Corollary 3.8. Let $X$ be a Banach space and $A$ a subset of $X^{*}$ that contains no copy of the basis of $\ell_{1}(\mathfrak{c})$. Then:

(1) For every $w^{*}$-compact subset $K \subset \overline{[A]}$ we have $\overline{\mathrm{co}} w^{*}(K)=\overline{\mathrm{co}}(K)$.

(2) Every convex subset $C \subset \overline{[A]}$ has 3 -control inside $X^{*}$.

Proof. First, observe that $\overline{[A]}$ contains no copy of the basis of $\ell_{1}(\mathfrak{c})$, because, if $\tau$ is a cardinal with cofinality $\operatorname{cf}(\tau)>\aleph_{0}$, then Talagrand proved in [20, Theorem 4] that $A$ contains a copy of the basis of $\ell_{1}(\tau)$ if and only if $\overline{[A]}$ has a copy of $\ell_{1}(\tau)$. Now it is enough to apply Proposition 3.5 and the fact that $\operatorname{cf}(\mathfrak{c})>\aleph_{0}($ see $[16$, p. 78$])$.

Corollary 3.9. Let $X$ be a Banach space and let $W$ be a subset of $X^{*}$ which is either weakly Lindelöf or is closed, convex and has the property $(C)$ of Corson. Then

(i) Every convex subset $C$ of $\overline{[W]}$ has 3 -control inside $X^{*}$.

(ii) For every $w^{*}$-compact subset $K$ of $\overline{[W]}$ we have $\overline{\mathrm{co}}^{w^{*}}(K)=\overline{\mathrm{co}}(K)$.

Proof. In both cases $W$ cannot contain a copy of the basis of $\ell_{1}(\mathfrak{c})$ and so (i) and (ii) follow from Corollary 3.8. Indeed, if $W$ is weakly Lindelöf, then 
$W$ fails to contain a copy of the basis of $\ell_{1}(\mathfrak{c})$ because such a copy would be a $w$-closed but non-w-Lindelöf subset.

Suppose now that $W$ is closed, convex and has the property $(C)$ of Corson. Recall that a closed convex subset $F$ of a Banach space has the property $(C)$ of Corson if $\bigcap_{i \in I} C_{i} \neq \emptyset$ whenever $\left\{C_{i}: i \in I\right\}$ is a family of closed convex subsets of $F$ with the countable intersection property, that is, $\bigcap_{i \in J} C_{i} \neq \emptyset$ for every countable subset $J \subset I$. If a closed convex subset $F$ of a Banach space has the property $(C)$ of Corson, then $F$ cannot contain a copy of the basis of $\ell_{1}(\mathfrak{c})$. Indeed, suppose $\mathcal{F}:=\left\{u_{\sigma}: \sigma<\mathfrak{c}\right\} \subset F$ is equivalent to the basis of $\ell_{1}(\mathfrak{c})$ and $C_{\sigma}:=\overline{\mathrm{co}}\left(\mathcal{F} \backslash\left\{u_{\sigma}\right\}\right)$. Clearly, the family $\left\{C_{\sigma}: \sigma<\mathfrak{c}\right\}$ has the countable intersection property but $\bigcap_{\sigma<\mathfrak{c}} C_{\sigma}=\emptyset$.

Remark 3.10. In [19, Problem 4.5] Talagrand asks, among other things, if $\overline{\mathrm{co}}^{*}(K)=\overline{\mathrm{co}}(K)$ whenever $K$ is a $w^{*}$-compact $w$-Lindelöf subset of a dual Banach space $X^{*}$. Cascales, Namioka and Vera proved in [5, Corollary E] (see also [4, Theorem 4.5]) that every $w^{*}$-compact $w$-Lindelöf subset of a dual Banach space $X^{*}$ is fragmented by the dual norm. So, applying [18, Theorem 2.3], they gave an affirmative answer to the question posed by Talagrand. Clearly, this result is a particular case of Proposition 3.5 because a $w$-Lindelöf subset cannot contain a copy of the basis of $\ell_{1}(\mathfrak{c})$, and so it does not have a $w^{*}-\mathbb{N}$-family.

\section{Convex subsets of Banach spaces with $w^{*}$-angelic closed dual} unit ball . If $Y$ is a Banach space, the closed dual unit ball $B\left(Y^{*}\right)$ is said to be $w^{*}$-angelic if given a subset $A$ of $B\left(Y^{*}\right)$ and $a \in \bar{A}^{w^{*}}$, there exists a sequence $\left\{a_{n}: n \geq 1\right\} \subset A$ such that $a_{n} \stackrel{w^{*}}{\rightarrow} a$. In this section we consider a particularly favorable case of the problem of the control of the distance $\widehat{d}\left(\overline{\mathrm{co}} w^{*}(K), C\right)$ by the distance $\widehat{d}(K, C), C$ being a convex subset of $X^{*}$ and $K$ a $w^{*}$-compact subset of $X^{*}$. This case appears when $C$ is a convex subset of some subspace $Y$ of $X^{*}$ such that the closed dual unit ball $\left(B\left(Y^{*}\right), w^{*}\right)$ is angelic. We prove that in this case there is 1-control.

Lemma 4.1. Let $K$ be a compact Hausdorff space with $\operatorname{card}(K) \geq 2, \mu$ a Radon measure on $K$ and $f \in C(K)$ a continuous real function on $K$. Let $\mu=\mu^{+}-\mu^{-}$be the decomposition of $\mu$ into its positive and negative parts. Then there exist distinct points $p_{1}, p_{2} \in K$ such that

$$
\left\|\mu^{+}\right\| f\left(p_{1}\right)-\left\|\mu^{-}\right\| f\left(p_{2}\right) \geq \mu(f) .
$$

Proof. Let $p_{1}, p_{2}$ be two distinct points of $K$ such that

$$
f\left(p_{1}\right)=\max \{f(p): p \in K\} \quad \text { and } \quad f\left(p_{2}\right)=\min \{f(p): p \in K\} .
$$

With this choice the statement holds because

$$
\mu^{+}(f)=\int_{K} f(k) d \mu^{+}(k) \leq \int_{K} f\left(p_{1}\right) d \mu^{+}(k)=\left\|\mu^{+}\right\| f\left(p_{1}\right),
$$




$$
\mu^{-}(f)=\int_{K} f(k) d \mu^{-}(k) \geq \int_{K} f\left(p_{2}\right) d \mu^{-}(k)=\left\|\mu^{-}\right\| f\left(p_{2}\right),
$$

whence $\left\|\mu^{+}\right\| f\left(p_{1}\right)-\left\|\mu^{-}\right\| f\left(p_{2}\right) \geq \mu^{+}(f)-\mu^{-}(f)=\mu(f)$.

If $I$ is an infinite set, let $\mathbf{c}(I)$ denote the subspace of $\ell_{\infty}(I)=C(\beta I)$ consisting of those elements which are constant on $I^{*}=\beta I \backslash I$.

Proposition 4.2. Let $I$ be an infinite set and $C$ a convex subset of $\mathbf{c}(I)$. Then for every $w^{*}$-compact subset $K$ of $\ell_{\infty}(I)\left(=\ell_{1}(I)^{*}\right)$ we have

$$
\widehat{d}\left(\overline{\mathrm{co}}^{w^{*}}(K), C\right)=\widehat{d}(K, C) .
$$

Proof. Let $K$ be a $w^{*}$-compact subset of $\ell_{\infty}(I)$. Without loss of generality (after a homothety if necessary), we suppose that $K \subset B\left(\ell_{\infty}(I)\right)$.

The trivial case. Assume that $K \subset \mathbf{c}(I)$. Observe that $\mathbf{c}(I)$ is Asplund (see $\left[9\right.$, p. 6]) because it is isomorphic to $\mathbf{c}_{0}(I)$. So, $\mathbf{c}(I)$ fails to contain a copy of $\ell_{1}(\mathfrak{c})$. Thus, from Proposition 3.5 we get $\overline{\mathrm{co}} w^{*}(K)=\overline{\mathrm{co}}(K)$ and so $\widehat{d}\left(\overline{\mathrm{co}} w^{*}(K), C\right)=\widehat{d}(\overline{\mathrm{co}}(K), C)=\widehat{d}(K, C)$.

The nontrivial case. Suppose that $K \backslash \mathbf{c}(I) \neq \emptyset$. This implies that $\widehat{d}(K, C)>0$. Assume that $\widehat{d}\left(\overline{\mathrm{co}} w^{*}(K), C\right)>\widehat{d}(K, C)$. Thus, for some $a, b>0$ we have

$$
\widehat{d}\left(\overline{\mathrm{co}}^{w^{*}}(K), C\right)>b>a>\widehat{d}(K, C) .
$$

Therefore, there exist vectors $w_{0} \in \overline{\mathrm{CO}}^{w^{*}}(K) \backslash \bar{C}$ and $\varphi \in S\left(\ell_{\infty}^{*}(I)\right.$ ) (see Lemma 2.1) such that inf $\varphi\left(w_{0}-C\right)>b$. Let $\varepsilon>0$ be such that $a+\varepsilon<b$. By the Riesz representation theorem (see $[17$, p. 46$]$ ) the dual $\ell_{\infty}^{*}(I)=C(\beta I)^{*}$ can be identified with the space of Radon Borel measures $M_{\mathrm{R}}(\beta I)$ on $\beta I$. On the other hand, if $\mu \in M_{\mathrm{R}}(\beta I)$, we have the decomposition $\mu=\mu_{1}+\mu_{2}$, where:

(i) $\mu_{1}=\left(\mu_{1 i}\right)_{i \in I} \in \ell_{1}(I)$ with $\mu_{1 i}=\mu(\{i\}), i \in I$, and $\mu_{2}=\mu\left\lceil I^{*}\right.$, that is, $\mu_{2}$ is the restriction of $\mu$ to the compact space $I^{*}$.

(ii) $\|\mu\|=\left\|\mu_{1}\right\|+\left\|\mu_{2}\right\|$.

So, $\ell_{\infty}^{*}(I)$ can be identified with the $\ell_{1}$-direct sum $\ell_{1}(I) \oplus_{1} M_{\mathrm{R}}\left(I^{*}\right)$, where $M_{\mathrm{R}}\left(I^{*}\right)$ is the space of Radon Borel measures on $I^{*}$. Thus, we have the decomposition $\varphi=\varphi_{1}+\varphi_{2}$ with $\varphi_{1} \in \ell_{1}(I), \varphi_{2} \in M_{\mathrm{R}}\left(I^{*}\right)$ and $1=\left\|\varphi_{1}\right\|+$ $\left\|\varphi_{2}\right\|$. Let $\varphi_{2}=\varphi_{2}^{+}-\varphi_{2}^{-}$be the decomposition of $\varphi_{2}$ into its positive and negative parts, and put $\lambda_{1}:=\left\|\varphi_{2}^{+}\right\|$and $\lambda_{2}:=\left\|\varphi_{2}^{-}\right\|$. Now we apply Lemma 4.1 to the compact space $I^{*}$, the Radon Borel measure $\varphi_{2}$ on $I^{*}$ and the continuous function $\check{w}_{0}$, where $\check{w}_{0}$ is the Stone-Čech continuous extension of $w_{0}$ to $\beta I$. So, there exist distinct points $p_{1}, p_{2} \in I^{*}$ such that

$$
\lambda_{1} \check{w}_{0}\left(p_{1}\right)-\lambda_{2} \check{w}_{0}\left(p_{2}\right) \geq \varphi_{2}\left(\check{w}_{0}\right) .
$$

Since $\check{w}_{0}$ is continuous on $\beta I$, there exist two infinite disjoint subsets $V_{1}, V_{2}$ of $I$ such that 
(i) $p_{i} \in \bar{V}_{i}^{\beta I}, i=1,2$.

(ii) For every $v_{i} \in \bar{V}_{i}^{\beta I}, i=1,2$,

$$
\lambda_{1} \check{w}_{0}\left(v_{1}\right)-\lambda_{2} \check{w}_{0}\left(v_{2}\right)>\varphi_{2}\left(\check{w}_{0}\right)-\varepsilon / 2 .
$$

Since $V_{1}, V_{2}$ are infinite disjoint subsets of $I$, we can choose two sequences of pairwise distinct points $\left\{d_{n}: n \geq 1\right\} \subset V_{1}$ and $\left\{e_{n}: n \geq 1\right\} \subset V_{2}$. Obviously,

$$
\lambda_{1} \check{w}_{0}\left(d_{n}\right)-\lambda_{2} \check{w}_{0}\left(e_{m}\right)>\varphi_{2}\left(\check{w}_{0}\right)-\varepsilon / 2, \quad \forall m, n \in \mathbb{N} .
$$

Let $\mu$ be a Radon Borel probability on $K$ with $r(\mu)=w_{0}$. Define the linear mapping $T_{n}: \ell_{\infty}(I) \rightarrow \mathbb{R}$ by $T_{n}(f)=\varphi_{1}(f)+\lambda_{1} f\left(d_{n}\right)-\lambda_{2} f\left(e_{n}\right)$ for every $n \in \mathbb{N}$ and every $f \in \ell_{\infty}(I)$. Clearly, $T_{n}$ is $\|\cdot\|$-continuous and $w^{*}$-continuous with $\left\|T_{n}\right\| \leq 1$. By (4.1) for every $n \geq 1$ we have

$$
\begin{aligned}
\varphi\left(w_{0}\right)-\varepsilon / 2 & =\varphi_{1}\left(w_{0}\right)+\varphi_{2}\left(\check{w}_{0}\right)-\varepsilon / 2 \\
& <\varphi_{1}\left(w_{0}\right)+\lambda_{1} \check{w}_{0}\left(d_{n}\right)-\lambda_{2} \check{w}_{0}\left(e_{n}\right)=T_{n}\left(w_{0}\right),
\end{aligned}
$$

whence

$$
\varphi\left(w_{0}\right)-\varepsilon / 2<T_{n}\left(w_{0}\right)=T_{n}(r(\mu))=\int_{K} T_{n}(f) d \mu(f) .
$$

Let $A_{n}:=\left\{f \in K: T_{n}(f)>\varphi\left(w_{0}\right)-\varepsilon\right\}$ for all $n \geq 1$. Observe that $A_{n}$ is a relatively $w^{*}$-open subset of $K$ for all $n \geq 1$.

Claim 1. $\mu\left(A_{n}\right) \geq \varepsilon / 2$ for all $n \geq 1$.

Indeed, for every $n \geq 1$ we have

$$
\begin{aligned}
\varphi\left(w_{0}\right)-\varepsilon / 2 & <T_{n}\left(w_{0}\right)=\int_{K} T_{n}(f) d \mu(f)=\left(\int_{A_{n}}+\int_{K \backslash A_{n}}\right) T_{n}(f) d \mu(f) \\
& \leq \mu\left(A_{n}\right)+\varphi\left(w_{0}\right)-\varepsilon .
\end{aligned}
$$

Thus $\mu\left(A_{n}\right) \geq \varepsilon / 2$ for all $n \geq 1$.

Let $B_{n}:=\bigcup_{m>n} A_{m}$ for every $n \geq 1$. The sequence $\left\{B_{n}\right\}_{n \geq 1}$ is decreasing and satisfies $\mu\left(B_{n}\right) \geq \varepsilon / 2$ for every $n \geq 1$. Hence $\mu\left(\bigcap_{n>1} B_{n}\right) \geq \varepsilon / 2$ and so $\bigcap_{n \geq 1} B_{n} \neq \emptyset$. Choose $g \in \bigcap_{n \geq 1} B_{n}$ and, inductively, the sequence $\left\{A_{n_{i}}\right\}_{i \geq 1}, n_{i}<n_{i+1}$, such that $g \in A_{n_{i}}$ for every $i \geq 1$. Then

$$
\varphi_{1}(g)+\lambda_{1} g\left(d_{n_{i}}\right)-\lambda_{2} g\left(e_{n_{i}}\right)=T_{n_{i}}(g)>\varphi\left(w_{0}\right)-\varepsilon, \quad \forall i \geq 1 .
$$

By a compactness argument, we can choose two distinct points $q_{1} \in$ ${\overline{\left\{d_{n_{i}}: i \geq 1\right\}}}^{\beta I} \backslash I \subset{\overline{V_{1}}}^{\beta I}$ and $q_{2} \in{\overline{\left\{e_{n_{i}}: i \geq 1\right\}}}^{\beta I} \backslash I \subset{\overline{V_{2}}}^{\beta I}$ such that

$$
\varphi_{1}(g)+\lambda_{1} \check{g}\left(q_{1}\right)-\lambda_{2} \check{g}\left(q_{2}\right) \geq \varphi\left(w_{0}\right)-\varepsilon .
$$

Let $\psi:=\varphi_{1}+\left(\lambda_{1} \delta_{q_{1}}-\lambda_{2} \delta_{q_{2}}\right)$. Observe that $\psi$ belongs to $S\left(\ell_{\infty}^{*}(I)\right)$.

Claim 2. $\inf \psi(g-C) \geq b-\varepsilon$.

Indeed, if $c \in C$ then $c \in \mathbf{c}(I)$ and so $\check{c}$ is constant on $I^{*}$. Thus

$$
\begin{aligned}
\psi(c) & =\varphi_{1}(c)+\left(\lambda_{1} \delta_{q_{1}}-\lambda_{2} \delta_{q_{2}}\right)(\check{c})=\varphi_{1}(c)+\left(\lambda_{1}-\lambda_{2}\right) \check{c}\left(q_{1}\right) \\
& =\varphi_{1}(c)+\varphi_{2}(\check{c})=\varphi(c) .
\end{aligned}
$$


So, taking into account (4.2) and the fact that inf $\varphi\left(w_{0}-C\right)>b$, for every $c \in C$ we have

$$
\begin{aligned}
\langle\psi, g-c\rangle & =\varphi_{1}(g)+\left(\lambda_{1} \check{g}\left(q_{1}\right)-\lambda_{2} \check{g}\left(q_{2}\right)\right)-\varphi(c) \\
& \geq \varphi\left(w_{0}\right)-\varepsilon-\varphi(c)=\left\langle\varphi, w_{0}-c\right\rangle-\varepsilon>b-\varepsilon .
\end{aligned}
$$

Therefore, we get $d(g, C) \geq b-\varepsilon$. On the other hand, as $g \in K$, we have $d(g, C)<a$ by hypothesis. So $b-\varepsilon<a$, which contradicts the choice of $\varepsilon$ and completes the proof.

Proposition 4.3. Let $X$ be a Banach space and $Y$ a closed subspace of $X^{*}$ with $w^{*}$-angelic closed dual unit ball $\left(B\left(Y^{*}\right), w^{*}\right)$. If $C$ is a convex subset of $Y$, then $\widehat{d}\left(\overline{\mathrm{co}} w^{*}(K), C\right)=\widehat{d}(K, C)$ for every $w^{*}$-compact subset $K$ of $X^{*}$. Moreover, $\overline{\mathrm{co}} w^{*}(K)=\overline{\mathrm{co}}(K)$ for every $w^{*}$-compact subset $K$ of $Y$.

Proof. Let $C$ be a convex subset of $Y$ and suppose that there exist a $w^{*}$-compact subset $K \subset B\left(X^{*}\right)$ and $0<a, b<1$ such that

$$
\widehat{d}\left(\overline{\mathrm{co}} w^{*}(K), C\right)>b>a>\widehat{d}(K, C) .
$$

Let $w_{0} \in \overline{\mathrm{co}^{*}} w^{*}(K)$ be such that $d\left(w_{0}, C\right)>b$. By Lemma 2.1 there exists $\varphi_{0} \in S\left(X^{* *}\right)$ such that $\inf \varphi_{0}\left(w_{0}-C\right)>b$. Let $\varepsilon>0$ be such that $b+\varepsilon<$ $\inf \varphi_{0}\left(w_{0}-C\right)$ and define

$$
\begin{aligned}
& U:=\left\{\varphi \in B\left(X^{* *}\right):\left\langle\varphi, w_{0}\right\rangle \geq\left\langle\varphi_{0}, w_{0}\right\rangle-\varepsilon\right\}, \\
& V:=\left\{x \in B(X):\left\langle w_{0}, x\right\rangle \geq\left\langle\varphi_{0}, w_{0}\right\rangle-\varepsilon\right\} .
\end{aligned}
$$

Observe that $\varphi_{0} \in U$ and, as $\left\langle\varphi_{0}, w_{0}\right\rangle-\varepsilon<1$, also $U=\bar{V}^{w^{*}}$. If $i: Y \rightarrow X^{*}$ is the canonical inclusion, then $i^{*}: X^{* *} \rightarrow Y^{*}$ satisfies $i^{*}\left(\varphi_{0}\right) \in i^{*}(U)=$ $\bar{i}^{*}(V) w^{*} \subset B\left(Y^{*}\right)$. Since $\left(B\left(Y^{*}\right), w^{*}\right)$ is angelic, there exists a sequence $\left\{x_{n}\right.$ : $n \geq 1\} \subset V$ such that $i^{*}\left(x_{n}\right) \stackrel{w^{*}}{\rightarrow} i^{*}\left(\varphi_{0}\right)$ in the $w^{*}$-topology $\sigma\left(Y^{*}, Y\right)$. Let $T: X^{*} \rightarrow \ell_{\infty}$ be the continuous linear mapping such that $T(u)=\left(u\left(x_{n}\right)\right)_{n \geq 1}$ for all $u \in X^{*}$. Then:

(1) $\|T\| \leq 1$ and, moreover, $T$ is $w^{*}-w^{*}$-continuous on bounded subsets of $X^{*}$.

(2) As $i^{*}\left(x_{n}\right) \stackrel{w^{*}}{\rightarrow} i^{*}\left(\varphi_{0}\right)$, for every $y \in Y$ we have $y\left(x_{n}\right)=i^{*}\left(x_{n}\right)(y) \rightarrow$ $i^{*}\left(\varphi_{0}\right)(y)$. Hence $T(Y) \subset \mathbf{c}(\mathbb{N})=\left\{f \in \ell_{\infty}: \check{f}\left\lceil\mathbb{N}^{*}\right.\right.$ is constant $\}$.

Let $\tilde{C}:=T(C), T(K)=: H \subset B\left(\ell_{\infty}\right)$ and $v_{0}:=T\left(w_{0}\right)$. Clearly, $H$ is a $w^{*}$-compact subset of $B\left(\ell_{\infty}\right)$ such that $\widehat{d}(H, \tilde{C}) \leq \widehat{d}(K, C)<a$ because $\|T\| \leq 1$, and $v_{0} \in \overline{\mathrm{co}} w^{*}(H)$. Let $e_{n}: \ell_{\infty} \rightarrow \mathbb{R}, n \geq 1$, be the $n$th canonical projection. Then $\left\{e_{n}: n \geq 1\right\} \subset B\left(\ell_{\infty}^{*}\right)$ and $T^{*}\left(e_{n}\right)=x_{n}, n \geq 1$. Let $\eta_{0}$ be a $w^{*}$-cluster point of $\left\{e_{n}: n \geq 1\right\}$ in $B\left(\ell_{\infty}^{*}\right)$. Clearly, $\eta_{0} \in S\left(\ell_{\infty}^{*}\right)$.

Claim. $\inf \eta_{0}\left(v_{0}-\tilde{C}\right) \geq b$.

Indeed, first $T^{*}\left(\eta_{0}\right)$ is a $w^{*}$-cluster point of $\left\{x_{n}: n \geq 1\right\}$ in $B\left(X^{* *}\right)$. Thus

(i) $T^{*}\left(\eta_{0}\right) \in U$, whence $\left\langle T^{*}\left(\eta_{0}\right), w_{0}\right\rangle \geq\left\langle\varphi_{0}, w_{0}\right\rangle-\varepsilon$. 
(ii) If $c \in C$, for every $n \geq 1$ we have $\left\langle T^{*}\left(e_{n}\right), c\right\rangle=\left\langle x_{n}, c\right\rangle \rightarrow\left\langle\varphi_{0}, c\right\rangle$, whence $\left\langle T^{*}\left(\eta_{0}\right), c\right\rangle=\left\langle\varphi_{0}, c\right\rangle$. Therefore, for every $c \in C$ we have

$$
\begin{aligned}
\left\langle\eta_{0}, v_{0}-T c\right\rangle & =\left\langle\eta_{0}, T w_{0}-T c\right\rangle=\left\langle T^{*}\left(\eta_{0}\right), w_{0}-c\right\rangle \\
& \geq\left\langle\varphi_{0}, w_{0}\right\rangle-\varepsilon-\left\langle\varphi_{0}, c\right\rangle \\
& =\left\langle\varphi_{0}, w_{0}-c\right\rangle-\varepsilon>b+\varepsilon-\varepsilon=b
\end{aligned}
$$

and this proves the Claim.

Therefore we get $d\left(v_{0}, \tilde{C}\right) \geq b$. On the other hand, from Proposition 4.2 we deduce that $d\left(v_{0}, \tilde{C}\right)<a$. So, we get a contradiction and this proves that $\widehat{d}\left(\overline{\mathrm{co}} w^{*}(K), C\right)=\widehat{d}(K, C)$. Finally, if $K$ is a $w^{*}$-compact subset of $Y$, taking $C=\overline{\mathrm{co}}(K)$ in the above argument, we deduce that $\overline{\mathrm{co}} w^{*}(K)=\overline{\mathrm{co}}(K)$.

Corollary 4.4. Let $X$ be a Banach space and $W$ a $w^{*}$-closed $w$-Lindelöf subset of $X^{*}$. Then $C$ has 1-control inside $X^{*}$ whenever $C$ is a convex subset of $\overline{[W]}$.

Proof. Let $W_{n}:=\{z \in W:\|z\| \leq n\}$ for all $n \geq 1$. Then $W_{n}$ is a $w^{*}$-compact $w$-Lindelöf subset of $X^{*}$ and $\left[\overline{\left.W_{n}\right]}\right.$ is WLD (weakly Lindelöf determined) by $[4$, Corollary 6.4]. Therefore $\overline{[W]}$ is WLD because $\overline{[W]}=$ $\overline{\bigcup_{n \geq 1}\left[\overline{\left.W_{n}\right]}\right.}$ (for instance, apply $[1$, Theorem 1.6]) and so the closed unit ball of $\overline{[W]}^{*}$ is $w^{*}$-angelic. Now apply Proposition 4.3.

Acknowledgements. The authors are grateful to the referee who made numerous remarks and suggestions which helped to improve this paper.

\section{References}

[1] S. Argyros and S. Mercourakis, On weakly Lindelöf Banach spaces, Rocky Mountain J. Math. 23 (1993), 395-446.

[2] B. Balcar and F. Franěk, Independent families in complete Boolean algebras, Trans. Amer. Math. Soc. 274 (1982), 607-618.

[3] B. Cascales, W. Marciszewski and M. Raja, Distance to spaces of continuous functions, Topology Appl. 153 (2006), 2303-2319.

[4] B. Cascales, I. Namioka and J. Orihuela, The Lindelöf property in Banach spaces, Studia Math. 154 (2003), 165-192.

[5] B. Cascales, I. Namioka and G. Vera, The Lindelöf property and fragmentability, Proc. Amer. Math. Soc. 128 (2000), 3301-3309.

[6] G. Choquet, Lectures on Analysis. Vol. II, W. A. Benjamin, New York, 1969.

[7] W. W. Comfort and S. Negrepontis, Chain Conditions in Topology, Cambridge Tracts in Math. 79, Cambridge Univ. Press, 1982.

[8] J. Diestel, Sequences and Series in Banach Space, Springer, New York, 1984.

[9] M. Fabian, Gâteaux Differentiability of Convex Functions and Topology. Weak Asplund Spaces, CMS Ser. Monogr. Adv. Texts, Wiley-Interscience, New York, 1997.

[10] M. Fabian, P. Hájek, V. Montesinos and W. Zizler, A quantitative version of Krein's theorem, Rev. Mat. Iberoamericana 21 (2005), 237-248. 
[11] A. S. Granero, An extension of the Krein-Šmulian theorem, ibid. 22 (2006), 93-110.

[12] A. S. Granero, P. Hájek and V. Montesinos, Convexity and $w^{*}$-compactness in Banach spaces, Math. Ann. 328 (2004), 625-631.

[13] A. S. Granero and M. Sánchez, The class of universally Krein-Šmulian Banach spaces, Bull. London Math. Soc. 39 (2007), 529-540.

[14] - - - Convexity, compactness and distances, in: Methods in Banach Space Theory, London Math. Soc. Lecture Note Ser. 337, J. M. F. Castillo and W. B. Johnson (eds.), Cambridge Univ. Press, 2006, 215-237.

[15] R. Haydon, Some more characterizations of Banach spaces containing $\ell_{1}$, Math. Proc. Cambridge Philos. Soc. 80 (1976), 269-276.

[16] I. Juhász, Cardinal Functions in Topology, Math. Centre Tracts 34, Amsterdam, 1971.

[17] H. E. Lacey, The Isometric Theory of Classical Banach Spaces, Springer, Berlin, 1974 .

[18] I. Namioka, Radon-Nikodym compact spaces and fragmentability, Mathematika 34 (1989), 258-281.

[19] M. Talagrand, Deux généralisations d'un théorème de I. Namioka, Pacific J. Math. 81 (1979), 239-251.

[20] - Sur les espaces de Banach contenant $\ell_{1}(\tau)$, Israel J. Math. 40 (1981), 324-330.

[21] R. C. Walker, The Stone-Čech Compactification, Springer, Berlin, 1974.

Departamento de Análisis Matemático

Facultad de Matemáticas

Universidad Complutense de Madrid

28040 Madrid, Spain

E-mail: AS_granero@mat.ucm.es

msam0003@encina.pntic.mec.es

Received September 27, 2006

Revised version July 19, 2007 